\title{
Breast cancer survival prediction using seven prognostic biomarker genes
}

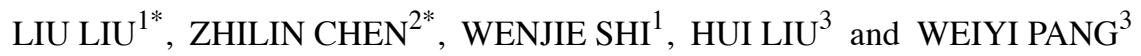 \\ ${ }^{1}$ Department of Pharmacy, Pharmacy School of Guilin Medical University, Guilin, Guangxi 541000; \\ ${ }^{2}$ Department of Breast and Thoracic Oncological Surgery, The First Affiliated Hospital of Hainan Medical College, \\ Haikou, Hainan 570102; ${ }^{3}$ School of Public Health, Guilin Medical University, Guilin, Guangxi 541000, P.R. China
}

Received February 12, 2019; Accepted June 13, 2019

DOI: $10.3892 / 01.2019 .10635$

\begin{abstract}
Breast cancer (BC) is one of the most prevalent forms of cancer globally. However, the practical relevance of the RNA expression-based prediction of BC is not clearly understood and requires further study. Using gene expression data downloaded from The Cancer Genome Atlas (TCGA), a risk score staging classification was created using Cox's multiple regression and was used to predict the clinical outcomes of patients with BC. In total, 7 genes, including AC123595.1, leukocyte immunoglobulin-like receptor B5, CD209 molecule, AL049749.1, lymphatic vessel endothelial hyaluronan receptor 1, transmembrane protein 190 and tubulin $\alpha$ 3D chain were identified in association with patient survival. The patients with lower risk scores had considerably improved survival rates than those with higher risk scores. Compared with other clinical factors, the risk score more accurately predicted the clinical outcome of patients with BC. In summary, 7 genes were identified using the Cox regression model, and subsequently used to develop a risk staging model for $\mathrm{BC}$, which may be of use for the medical management of patients.
\end{abstract}

\section{Introduction}

Breast cancer (BC), a type of cancer most frequently diagnosed in females, is a considerable threat to female health worldwide. In the USA, 230,000 new cases of BC are diagnosed each year, of which $\sim 5.6 \%$ are women $>40$ years old (1). Although the surgical methods and drug regimens used to treat $\mathrm{BC}$ are constantly improving, the clinical outcomes of individual patients remain difficult to predict due, in part, to a number of

Correspondence to: Lecturer Hui Liu or Professor Weiyi Pang, School of Public Health, Guilin Medical University, 1 Zhiyuan Road, Guilin, Guangxi 541000, P.R. China

E-mail: huihuiabcd@126.com

E-mail: p.weiyi@live.cn

*Contributed equally

Key words: breast cancer, prognostic signature, Cox regression model clinically associated factors (2,3). In previous studies, tumor size, tissue grade and lymph node status have been used to speculate the clinical outcomes of patients $(4,5)$. However, research has suggested that the accuracy of these indicators is not satisfactory (6). As a consequence of the development of sequencing technologies, the search for novel biomarkers has rapidly accelerated (7). The expression levels of specific microRNAs (miRNAs/miRs) have been identified as potential biomarkers for predicting survival rate in several types of human cancer. Han et al (8) revealed that the upregulation of miR-21 was associated with aggressive advancement and poor prognosis in patients with cervical cancer. Recent findings have reported that miR-106b-5p activity may be used to classify tumor protein 53-like bladder tumors into more- and less-favorable predictive categories (9). Similarly, Yue et al (10) revealed that serum miR-205 may be a useful biomarker for the diagnosis of glioma, and a predictive factor for gliomas of an advanced pathological grade. In addition, the expression levels of several other RNAs have been indicated as predictors of survival, including cohesin subunit SA-2 in bladder cancer and high mobility group protein B1 in lung adenocarcinoma $(11,12)$. Furthermore, long non-coding RNAs (lncRNAs) HOXA distal transcript antisense RNAand HOX transcript antisense RNA have been used as novel biomarkers in the diagnosis of renal cell carcinoma and $\mathrm{BC}$, respectively $(13,14)$.

The findings of the aforementioned studies support the long-standing use of gene biomarkers in the clinical prediction of disease course and outcome. However, in these studies, predictions were based on single-gene biomarkers, which are known to be less reliable for predicting patient survival than their multi-gene counterparts (15). Furthermore, multi-gene indicators may enhance the sensitivity and specificity of prognosis for tumor patients when compared with those generated using single biomarker methods. In 2002, van de Vijver et al (16) reported the gene-expression profile to be a strong projector of disease stage in young patients with BC compared with clinical and histological measures (15), and in 2006, Paik et al (17) revealed that a 21-gene recurrence score was able to predict the degree of chemotherapy success in patients with breast cancer. In addition, the results of a study by Wang et al (18) illustrated that histological grades 1 and 3 could be distinguished with high accuracy from gene expression levels, determined using RNA-sequencing in patients with breast cancer. 
In the present study, a Cox multiple regression model was used to assess gene expression in BC samples from The Cancer Genome Atlas (TCGA; http://www.tcga.org/). Patients with high risk scores reported shorter survival rates compared with those with low risk scores, a finding that was further validated using the training and complete test datasets. Moreover, the risk score is independent of other clinical variables, and performs better than clinical information to determine $\mathrm{BC}$ prognosis. Risk scores and other clinical factors were combined to develop a nomogram enabling the accurate and convenient prediction of the 5- and 10-year survival rates of patients with BC.

\section{Materials and methods}

Data sources and pre-processing. The data of 631 cases of BC were downloaded from TCGA breast cancer database (TCGA-Breast Invasive Carcinoma), and included 87 cases in the healthy control group and 544 cases in the cancer group. Differentially-expressed genes were screened according to the criteria of $\mathrm{P}<0.01$ and $\log 2$-fold change $>2$. All data analysis and min-max normalization was performed using Perl and $\mathrm{R}$ scripts. The integrity of the patients' RNA expression profiles and clinically relevant information (age, sex, stage and histological type) was an important prerequisite for the selection of patients. In addition, complete ER, PR information was also a necessary condition for enrollment. Patients who had previously been diagnosed with breast cancer or any other cancer were excluded.

Training data set: Survival analysis and Cox multiple regression model. Following the identification of differentially-expressed genes in cancerous and adjacent-healthy tissues (using the R package edge; http://www.bioconductor. org/packages/release/bioc/html/edge.html), 87 samples that lacked survival data were excluded from the datasets, and the remaining 544 patients with $\mathrm{BC}$ were screened for subsequent analysis. The 544 patients with $\mathrm{BC}$ were randomly divided into a training dataset $(n=365)$ and a test dataset $(n=179)$ using scripts written in $\mathrm{R}$ (Table $\mathrm{I}$ ). With the aim to establish a multi-gene biomarker model of prognosis, the training dataset was then screened for biomarker genes that were significantly associated with the prognosis of patients with BC. The specific steps used were as follows: The association between differentially-expressed genes and overall survival (OS) in patients with $\mathrm{BC}$ was determined using a univariate Cox proportional regression model. Genes for which $\mathrm{P}<0.001$ were defined as significantly associated with prognosis, and Cox multivariate analysis was subsequently performed for these genes. The proportional hazard assumption $(\mathrm{P}=0.806)$ was tested using Stata version 15.0 (https://www.stata.com) prior to Cox proportional regression analysis in the final multivariate model. Finally, a BC prognostic model was determined using stepwise regression. The $\mathrm{R}$ packages function, coxph and survival were used to construct a risk score staging model (https://cran.r-project.org/web/packages/survival/index.html). The risk score formula was as follows:

$$
\text { Risk score }=\sum_{i}^{n} \beta i_{\mathrm{x}} x i
$$

Where $\mathrm{n}$ indicates the number of prognostic genes screened, $\mathrm{i}$ refers to the relative expression of corresponding gene. $\beta$ is the coefficient of the individual gene and $x$ indicates the relative expression of the gene. If $\beta>0$, genes are negatively correlated with the survival time or survival rate, and if $\beta<0$, genes are considered to be protective. Patients were categorized into high- and low-risk groups according to the median risk score (0.95). The risk score of each patient was calculated using a gene-based risk score prediction model. Additionally, an OS curve was created using the $\mathrm{R}$ package survival. A 2-sided log-rank test was utilized to determine variations in survival among high- and low-risk patients. Receiver operating characteristic (ROC) analysis using the R package survival ROC (19) was used to evaluate the sensitivity and specificity of the gene-based prognostic model in predicting medical outcomes.

Authentication of the 7-gene signature for survival projection in the validation and entire datasets. The predicted performance of the 7 differential gene model was authenticated using both the validation set and the entire dataset. Patients in both datasets were grouped according to the cut-off values of the experimental groups, separating the 2 groups of data into highand low-risk categories, respectively. Kaplan-Meier survival curves were generated, and the log-rank test was performed to reveal alterations in survival time among patients in both datasets. The ROC curve was generated to assess the clinical predictive power of 7 differentially-expressed gene signatures in both datasets.

Development of a novel nomogram including risk scores. The Cox proportional hazards regression model was used to determine whether the risk-scoring model was an autonomous predictive factor for patients with BC. Stratified analysis was performed to verify whether the 7 differential gene characteristics were independent prognostic factors for patients with $\mathrm{BC}$, compared with other clinical variables. In addition, a nomogram was constructed using the risk scores, age, sex and primary tumor staging and visualized using the $\mathrm{R}$ package rms (20). The accuracy of the model was assessed using the C-index. All data analysis and processing were conducted using R software (version 3.4.2; www.r-project.org).

\section{Results}

Identification of survival-associated genes in the training dataset. To identify novel genetic biomarkers associated with the clinical outcomes of patients with $\mathrm{BC}$, a univariate Cox proportional hazard regression model was applied to differently-expressed genes in BC and healthy breast tissues. In total, 18 genes were found to be significantly associated with OS $(\mathrm{P}<0.001)$. These genes were subsequently subjected to stepwise multivariate Cox regression analysis. As illustrated in Table II, 7 independent genes were selected using step-wise multivariate Cox regression analysis, and a gene-based prognostic model was established to estimate the survival risk of patients using the following equation:

Risk score $=(-0.1735 \times$ TMEM190 $)+(-0.1510 \times$ AL049749.1 $)$ $+(-0.2924 \times$ AC123595.1) + (-0.1024 x TUBA3D $)+(0.1990 \times$ LYVE1 $)+(0.4676 \times$ LILRB5 $)+(0.1744 \times$ CD209) 
Table I. Clinical characteristics of the patients with breast cancer in each dataset.

\begin{tabular}{|c|c|c|c|c|}
\hline Covariate & Total & Training set & Testing set & P-value \\
\hline $\mathrm{N}$ & 544 & 365 & 179 & \\
\hline Risk score & & & & 0.051 \\
\hline Low & 265 & 167 & 98 & \\
\hline High & 279 & 198 & 81 & \\
\hline Age (years) & & & & 0.382 \\
\hline$\leq 65$ & 388 & 256 & 132 & \\
\hline$>65$ & 156 & 109 & 47 & \\
\hline Sex & & & & 0.395 \\
\hline Male & 6 & 5 & 1 & \\
\hline Female & 538 & 360 & 178 & \\
\hline Stage & & & & 0.826 \\
\hline I & 99 & 69 & 30 & \\
\hline II & 300 & 201 & 99 & \\
\hline III & 136 & 90 & 46 & \\
\hline IV & 9 & 5 & 4 & \\
\hline Histological type & & & & 0.592 \\
\hline Infiltrating ductal & 397 & 267 & 130 & \\
\hline Infiltrating lobular & 94 & 62 & 32 & \\
\hline Mixed & 21 & 12 & 9 & \\
\hline Others & 32 & 24 & 8 & \\
\hline Estrogen receptor & & & & 0.492 \\
\hline Negative & 122 & 85 & 37 & \\
\hline Positive & 422 & 280 & 142 & \\
\hline Progesterone receptor & & & & 0.958 \\
\hline Negative & 171 & 115 & 56 & \\
\hline Positive & 373 & 250 & 123 & \\
\hline
\end{tabular}

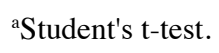

Table II. Seven prognostic genes significantly associated with overall survival in patients with breast cancer.

\begin{tabular}{lcccc}
\hline Gene name & Coefficient & Hazard ratio & 95\% confidence interval & P-value $^{\mathrm{a}}$ \\
\hline TMEM190 & -0.1735 & 0.8407 & $0.6531-1.0300$ & 0.05765 \\
lncRNA AL049749.1 & -0.1510 & 0.8598 & $0.5017-1.2051$ & 0.08750 \\
lncRNA AC123595.1 & -0.2924 & 0.7465 & $0.6183-0.9259$ & $0.04453^{\mathrm{b}}$ \\
TUBA3D & -0.1024 & 0.9027 & $0.6526-1.7124$ & 0.13771 \\
LYVE1 & 0.1990 & 1.2202 & $1.0347-1.5063$ & $0.04394^{\mathrm{b}}$ \\
LILRB5 & 0.4676 & 1.5962 & $1.3441-1.9163$ & $0.00084^{\mathrm{c}}$ \\
CD209 & 0.1744 & 1.1906 & $1.0275-1.4055$ & $0.04598^{\mathrm{b}}$ \\
\hline
\end{tabular}

${ }^{a}$ Multivariate Cox regression analysis. ${ }^{b} \mathrm{P}<0.05,{ }^{\mathrm{c}} \mathrm{P}<0.001$. TMEM190, transmembrane protein 190; TUBA3D, tubulin $\alpha$ 3d; LYVE1, lymphatic vessel endothelial hyaluronan receptor 1; LILRB5, leukocyte immunoglobulin like receptor B5; CD209, CD209 molecule.

Training dataset: Risk score performance. Final calculations indicated a median and mean risk score of 0.95 and 1.32 , respectively. The minimum and maximum values were 0.01 and 6.62, respectively. To confirm the performance of the risk score in predicting the survival rates of patients with $\mathrm{BC}$, the prognostic, 7-gene signature-based model was used to allocate a risk score for each patient. Patients were categorized as high-risk $(n=198)$ or low-risk $(n=167)$, where the median risk score was used as the cut-off value. Kaplan-Meier analysis revealed that the OS curves of these 2 groups 
A
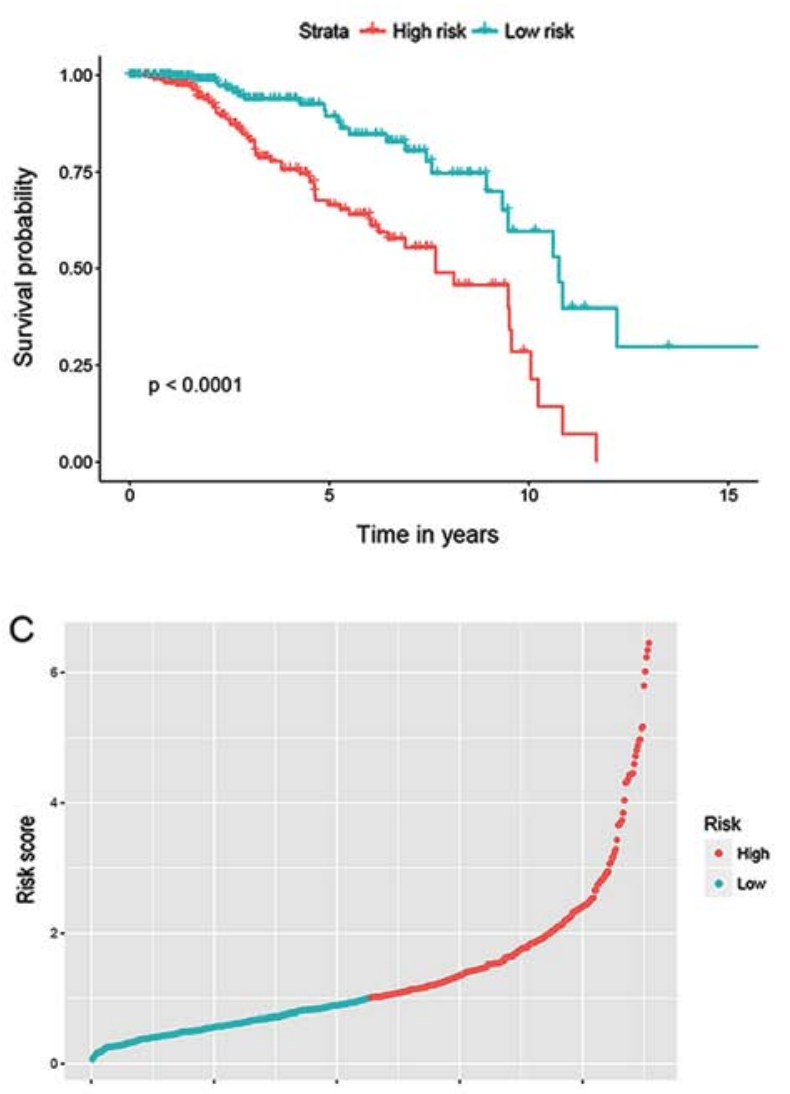

Number of patients
B
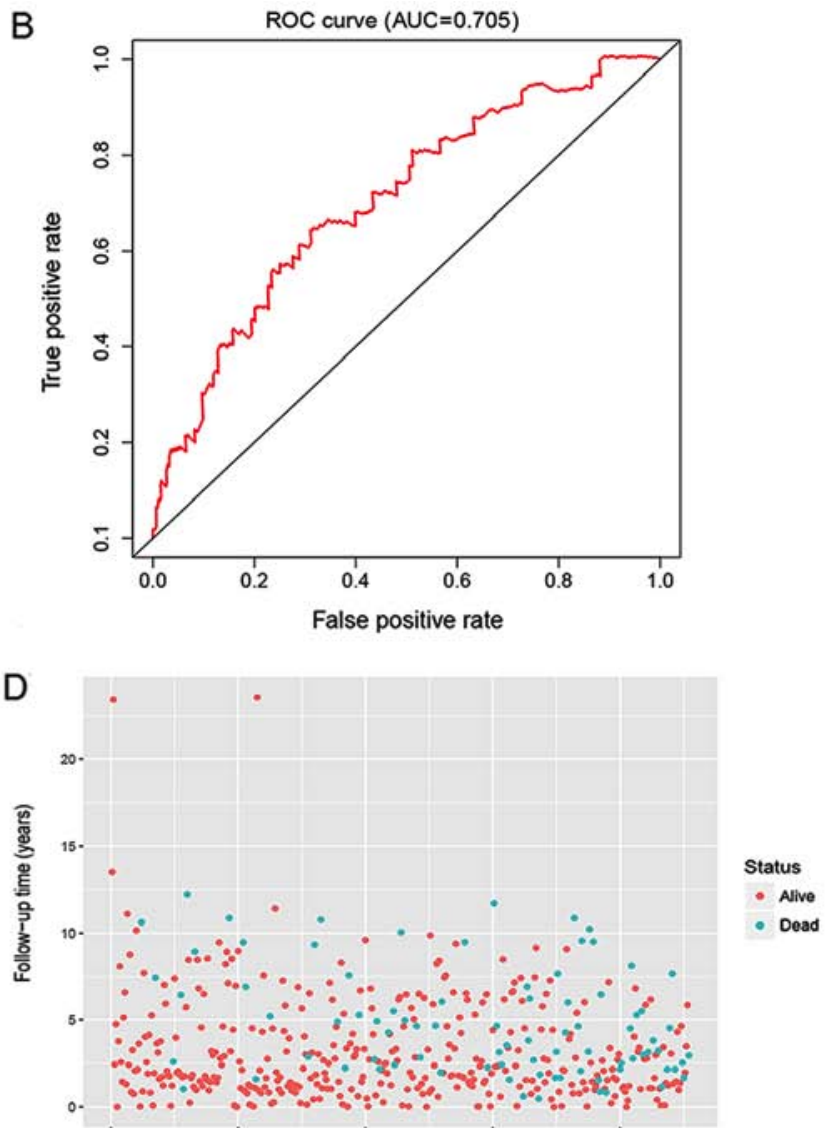

Number of patients

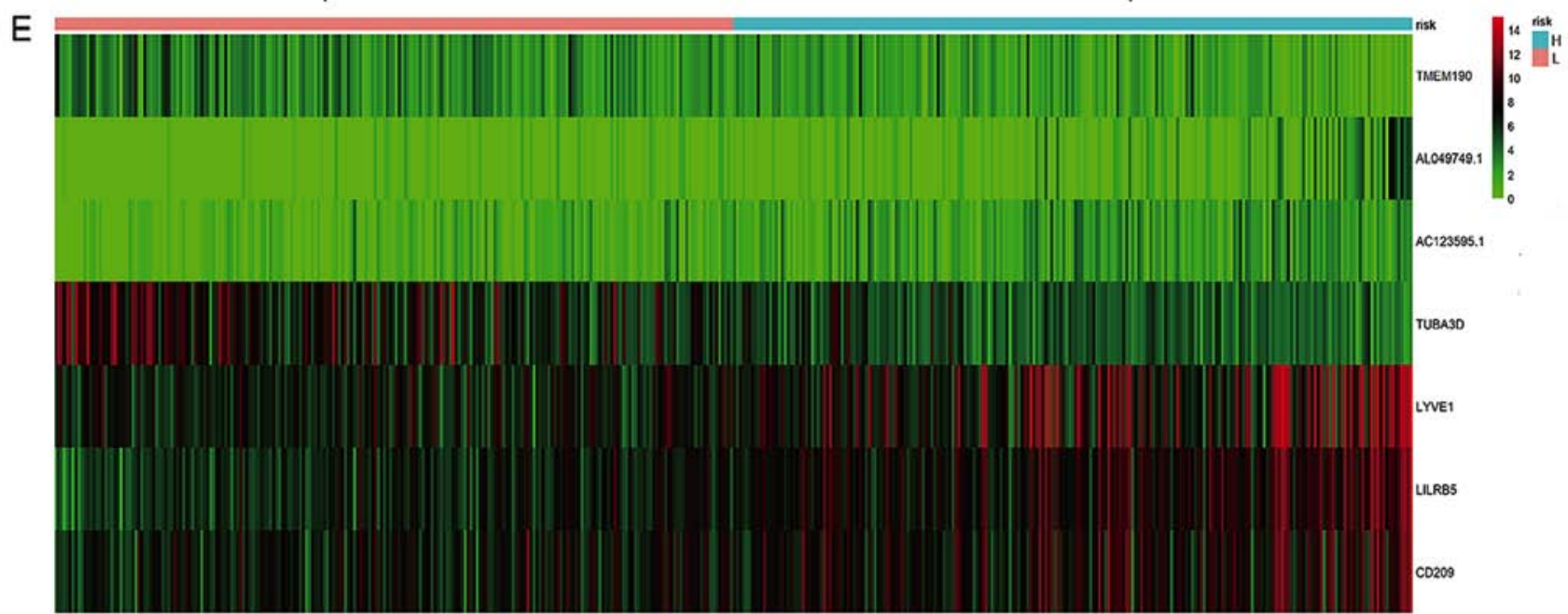

Figure 1. Performance of the 7-gene signature in the training data set. (A) Kaplan-Meier survival curve of overall survival between the high- and low-risk groups in the training data set. (B) ROC curves in the training data set. (C) Visualization of the cut-off value (0.95) of risk scores, the $\mathrm{x}$-axis represents number of patients. (D) The distribution of survival status in the training data set, the x-axis represents number of patients. (E) The expression profiles of patients in the training data set; green indicates reduced expression and red indicates increased expression (hierarchal clustering for the heatmap not shown). ROC, receiver operating characteristic; AUC, area under the curve; TMEM190, transmembrane protein 190; TUBA3D, tubulin $\alpha$ 3D chain; LYVE1, lymphatic vessel endothelial hyaluronan receptor 1; LILRB5, leukocyte immunoglobulin-like receptor B5; CD209, CD209 molecule.

were significantly different $(\mathrm{P}<0.001$; Fig. $1 \mathrm{~A})$. ROC curve analysis of the 10-year survival rate was used to evaluate the projection potential of the 7 genes. Moreover, the area under curve (AUC) for the 7-gene signature-based prognostic model was 0.705 at 120 months OS (Fig. 1B). The scattering of the risk score (Fig. 1C), survival status (Fig. 1D) and gene expression levels of the 7 genes (Fig. 1E) from each patient were also analyzed.
Validation of the performance of risk score in test datasets. To assess the strength of the prognostic model in patients with $\mathrm{BC}$, the risk scores in the test dataset $(\mathrm{n}=179)$ and the entire dataset $(n=544)$ were determined. In the test dataset, the patients were categorized into high-risk $(n=81)$ and low-risk $(n=98)$ groups per the risk-score model, and cut-off points were defined using the training dataset. Kaplan-Meier survival curves of the high- and low-risk groups were considerably 
A

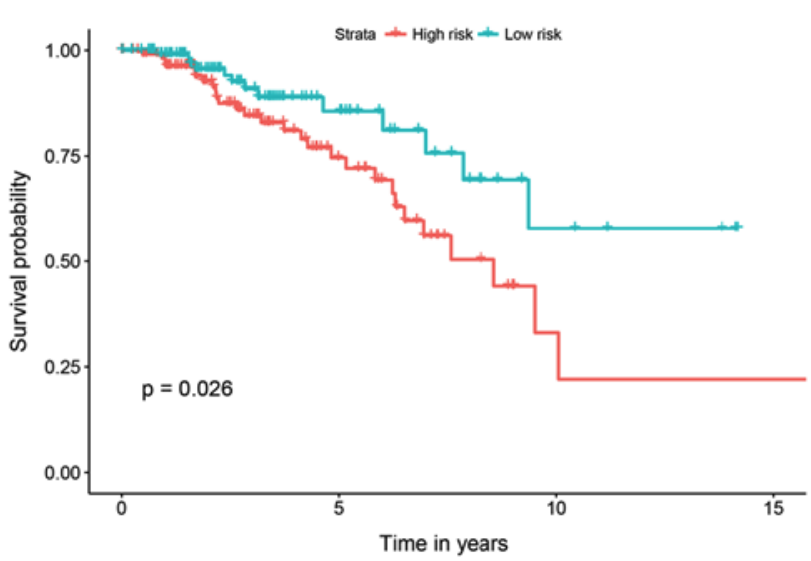

B

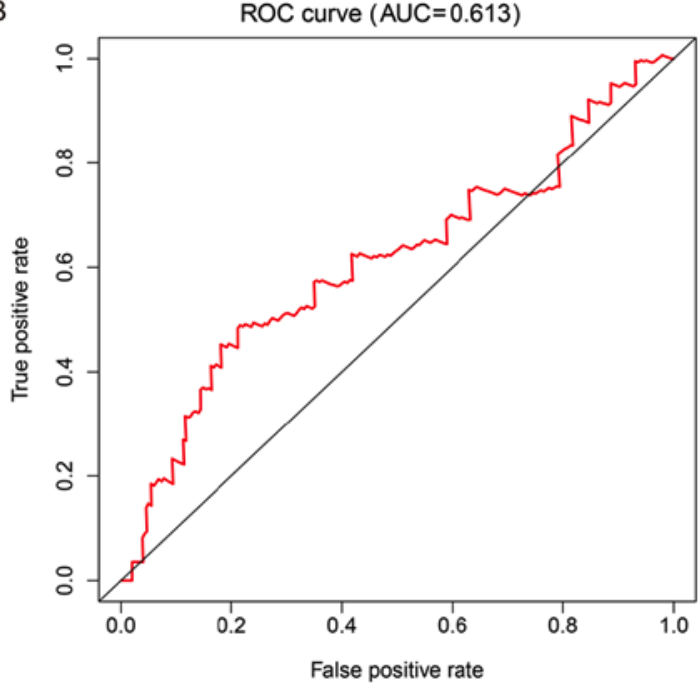

C

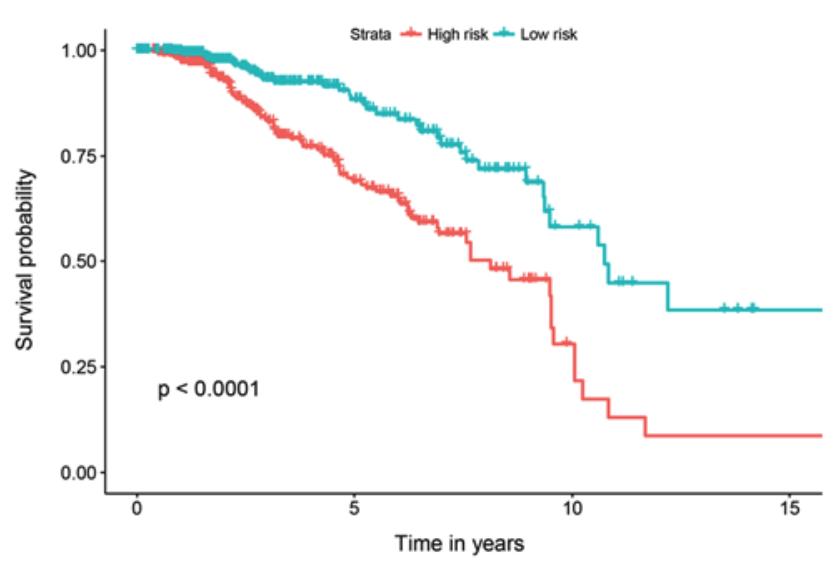

D

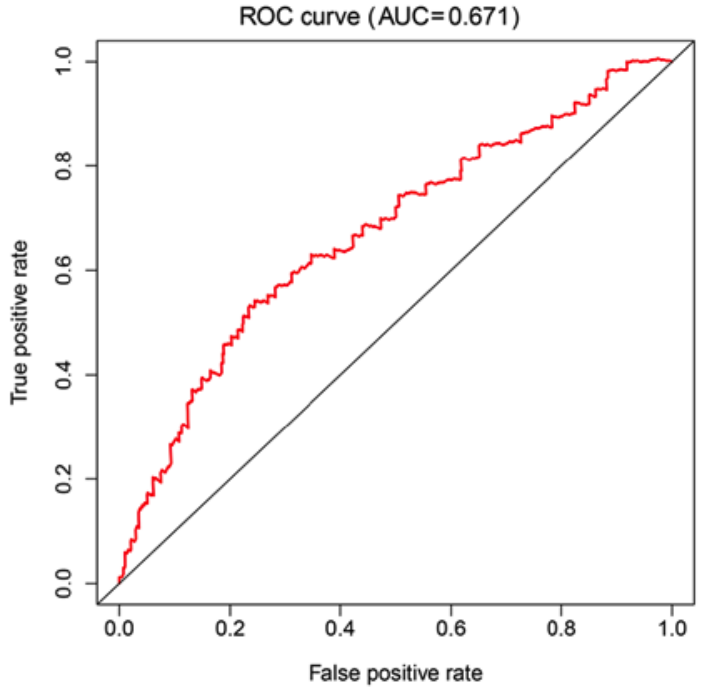

Figure 2. Performance of the risk score in the test and entire data sets. (A) The Kaplan-Meier survival curve of overall survival of patients with BC using the 7-gene signature in the test data set. (B) ROC curve analysis of the 7-gene signature in the test data set. (C) Kaplan-Meier survival curve analysis for overall survival of patients with $\mathrm{BC}$ using the 7-gene signature in the entire data set. (D) ROC curve analysis of the 7-gene signature in the entire data set. BC, breast cancer; ROC, receiver operating characteristic; AUC, area under the curve.

dissimilar in the test dataset. Compared with patients from the high-risk group, those from the low-risk group had significantly longer survival times ( $\mathrm{P}=0.026$; Fig. $2 \mathrm{~A})$. The AUC of the time-dependent ROC curves for the 7-gene signature in the test dataset was 0.613 at 10 years (Fig. 2B). When this signature was applied to the entire dataset, a conclusion was reached. Moreover, the 7-gene signature was used to classify patients in the entire TCGA dataset into high-risk $(n=279)$ and low-risk $(n=265)$ groups. The patients in the high-risk group exhibited shorter OS times compared with those in the low-risk group $(\mathrm{P}<0.0001$; Fig. $2 \mathrm{C})$. Authentication of the signature using all 544 patients generated a ROC AUC of 0.671 at 10 years (Fig. 2D). These outcomes suggested that the risk score was a robust predictor of clinical outcome for patients with BC. The distribution of the risk score model, survival status and gene expression patterns of patients in the test and entire datasets were also analyzed (Figs. 3 and 4). Fig. 3A shows that the cut-off value of the risk scores in the test data set is 0.95 . The distributions of survival status in the test data set is shown in Fig. 3B. Fig. 3C shows the expression profiles of patients in the test data set. LYYE1, LILRB5, CD209 was highly expressed in tumor tissues and TMEM190,
AC123595.1, AL049749.1 and TUBA3D expression was low in tumor tissues. The same conclusion was reached based on the data shown in Fig. 4.

Association between risk score and other clinical factors in the entire dataset. To determine the potential association between the risk score and clinical parameters [including age, sex, oestrogen receptor (Er) status, progesterone receptor $(\mathrm{Pr})$ status, tumor stage and histological type], multiple Cox hazard analyses were performed utilizing the entire dataset. As presented in Fig. 5, the risk score possessed a predictive ability separate from that of the other clinical parameters [hazard ratio (HR), 2.464; 95\% confidence interval (CI), 1.546-3.929; $\mathrm{P}<0.0010]$ (Fig. 5). This suggests that the prognostic capacity of the risk score was also independent of these other clinical variables.

Stratified analyses were conducted to determine whether the 7-gene signature held predictive importance. Patients were categorized into younger $(n=388)$ and elder $(n=256)$ strata depending on the median age (60 years); younger patients were subsequently divided into high-risk ( $\mathrm{n}=193)$ and low-risk $(n=195)$ groups. Patients in the low-risk group had significantly 


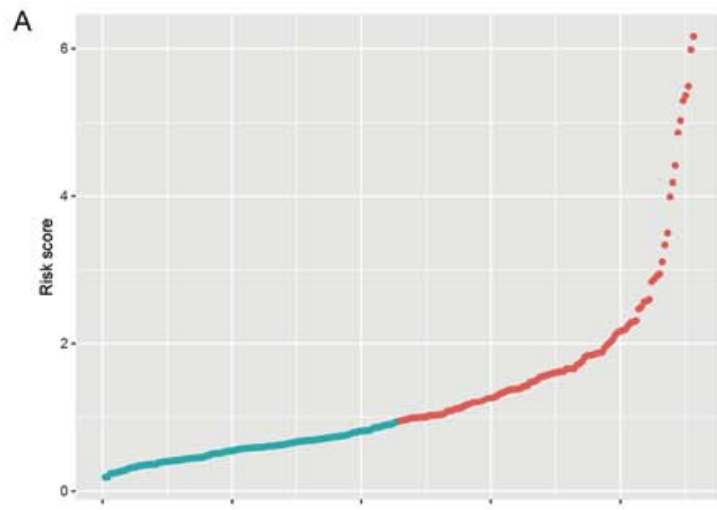

Number of patients

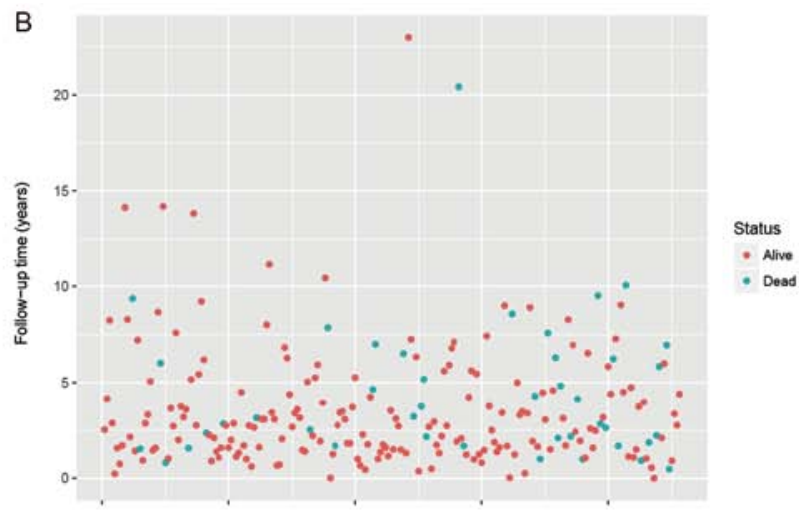

Number of patients

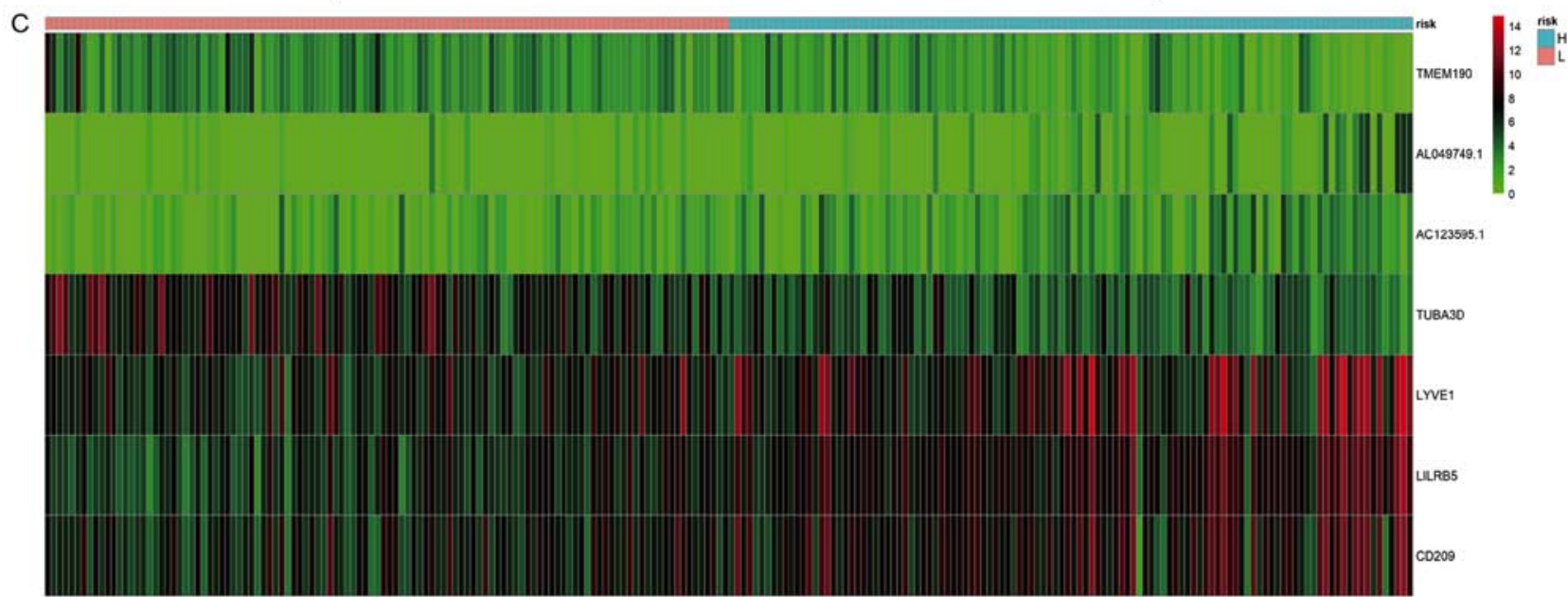

Figure 3. Seven-gene signature distributions, survival status and expression profiles of patients in the test data set. (A) Visualization of the cut-off value (0.95) of the risk scores in the test data set, the $\mathrm{x}$-axis represents number of patients. (B) The distributions of survival status in the test data set, the $\mathrm{x}$-axis represents number of patients. (C) The expression profiles of patients in the test data set, green indicate reduced expression and red indicate increased expression (hierarchal clustering for the heatmap not shown). TMEM190, transmembrane protein 190; TUBA3D, tubulin $\alpha$ 3D chain; LYVE1, lymphatic vessel endothelial hyaluronan receptor 1; LILRB5, leukocyte immunoglobulin-like receptor B5; CD209, CD209 molecule.

longer OS times compared with those in the high-risk group $(\mathrm{P}=0.0036$; Fig. 6A). Likewise, patients in the elder group were separated into low- and high-risk groups with different OS times $(\mathrm{P}=0.051$; Fig. $6 \mathrm{~B})$. The patients were concurrently categorized into early-stage $(n=399)$ and advanced-stage $(n=45)$ groups depending on the primary tumor stage. The early-stage patients were then divided into a high-risk group $(n=203)$ with shorter survival, and a low-risk group $(n=196)$ with an extended survival period ( $\mathrm{P}=0.0013$; Fig. 6C). Similarly, the advanced-stage patient group was divided into 2 risk subgroups with significantly different survival times $(\mathrm{P}=0.02$; Fig. 6D). The results of these analyses suggested that the 7-gene signature may function as an autonomous indicator of survival for patients with BC.

An innovative nomogram for the prediction of patient outcome. To complement the predictive capacity of the risk score, an innovative nomogram was developed to predict the prognosis of patients with BC. The nomogram was based on 6 projecting factors and comprised risk score, age, sex, Er status, Pr status, tumor stage and histological type. A high total score indicated low 5- and 10-year survival rates, whilst a low total score indicated improved survival rates. The $\mathrm{C}$-index of the nomogram for predicting OS was 0.755 (95\% CI, 0.719-0.791) in the main cohort. This suggested that in medical practice, the model was appropriate for predicting the outcomes of patients with BC (Fig. 7).

\section{Discussion}

A number of previously published studies have reported numerous individual prognostic biomarkers associated with $\mathrm{BC}$. Using reverse transcription-quantitative PCR and western blotting, Zhao et al (21) analyzed the expression of inosine monophosphate dehydrogenase 2(IMPDH2) in 40 matched normal and $\mathrm{BC}$ tissues, the results of which indicated that a high level of IMPDH2 expression was associated with poor patient outcome. Another study revealed that the expression level of lncRNA-AK058003 was upregulated in BC tissues compared with healthy adjacent tissue, and that this was also indicative of poor prognosis and associated with tumor cell invasion and metastasis (22) In addition, Guo et al (23) demonstrate that the upregulation of miR-1915-3p and downregulation of miR-455-3p in the serum of patients with BC promoted lymph node metastasis in patients with in situ carcinomas, compared with patients without lymph node metastasis. However, the aforementioned studies were based on the assessment of single gene biomarkers only, 

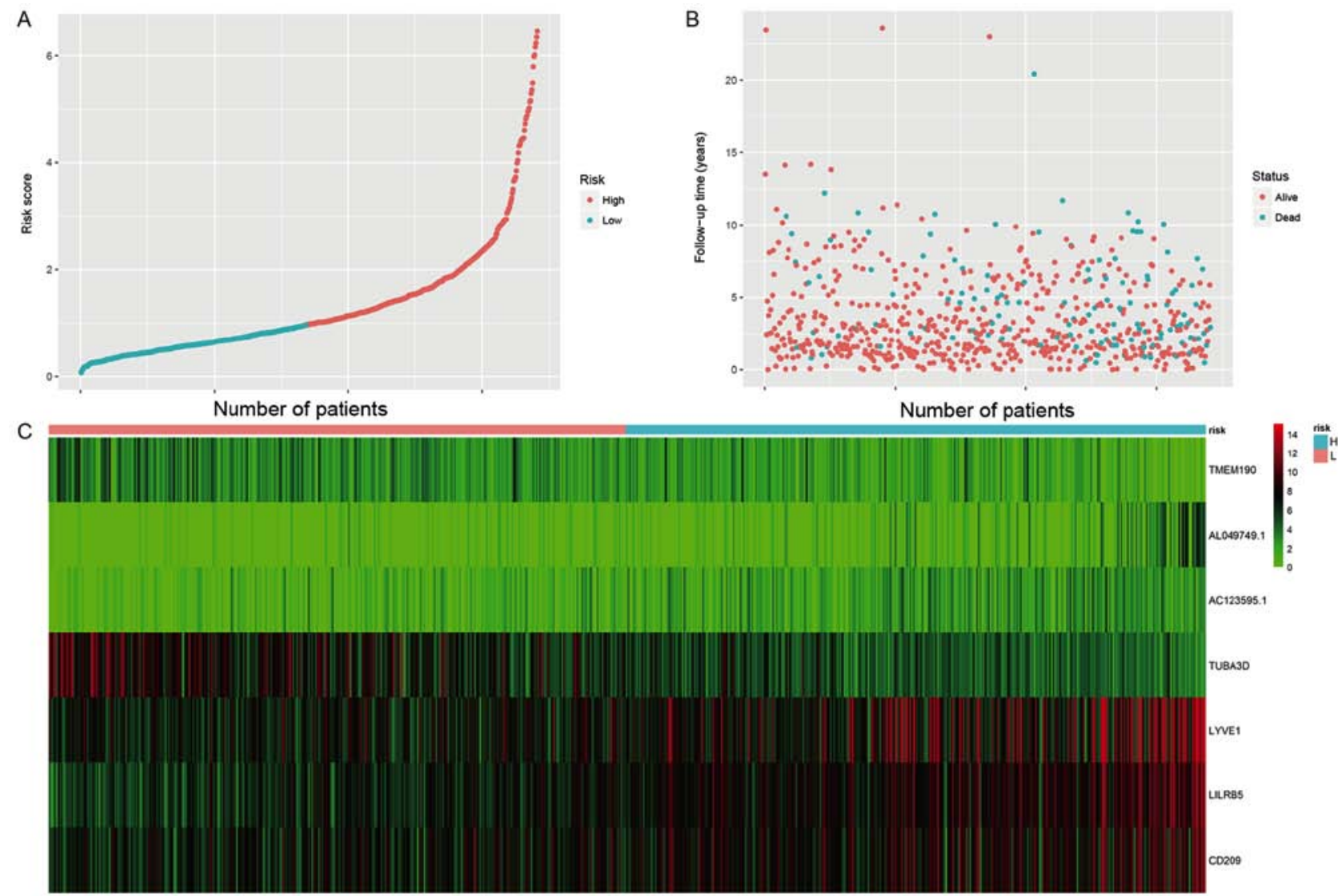

Figure 4. Seven-gene signature distributions, survival status and expression profiles of patients in the entire data set. (A) Visualization of the cut-off value (0.95) of risk scores in the entire data set, the x-axis represents number of patients. (B) The distributions of survival status in the entire data set, the $\mathrm{x}$-axis represents number of patients. (C) The expression profiles of patients in the entire data set, green indicates reduced expression and red indicates increased expression (hierarchal clustering for the heatmap not shown). TMEM190, transmembrane protein 190; TUBA3D, tubulin $\alpha$ 3D chain; LYVE1, lymphatic vessel endothelial hyaluronan receptor 1; LILRB5, leukocyte immunoglobulin-like receptor B5; CD209, CD209 molecule.

which when used as a prognostic standard, inevitably lead to clinical bias (16). Therefore, it is necessary to develop novel multi-gene models to predict the survival of patients with BC, and to establish personalized treatment programs. The use of multi-gene biomarkers increases the sensitivity and specificity of the predictive model, ultimately improving overall credibility (15).

In the present study, Cox multiple regression analysis of BC RNA-Sequencing data downloaded from TCGA was performed in order to identify genes associated with patient OS; 7 genes were found to meet the selection criteria. Survival analysis indicated that patients with high-risk scores possessed considerably shorter OS times than patients with low risk scores $(\mathrm{P}<0.001)$. The AUC of this model was 0.705 at 120 months OS, indicating that the predictive value of the 7-gene signature may be utilized for survival prediction. Compared with other specific medical parameters (including age, sex, tumor stage and histological type) risk scores were better predictors of patient survival, indicating that the 7-gene signature may be of value in further research. Additionally, in order to better adapt the multi-gene risk score model to current clinical requirements, other clinical factors were combined to develop a novel nomogram that could accurately and conveniently predict the 5 - and 10 -year survival rates of patients. The nomogram may be able to more accurately determine the correct course of treatment for patients with a low survival rate, in comparison to the traditional Tumor-Node-Metastasis classification systems or nomograms containing clinical features alone or which utilized only a single biomarker.

Among the 7 genes identified in the present study, lymphatic vessel endothelial hyaluronan receptor 1 (LYVE1), leukocyte immunoglobulin-like receptor B5 (LILRB5) and CD209 molecule (CD209) were risk-associated, demonstrating that the expression levels of these genes negatively correlated with $\mathrm{BC}$ survival time; conversely, the expression levels of AC123595.1, AL049749.1, transmembrane protein 190 and tubulin $\alpha$-3D chain were positively associated with survival. LYVE1 and CD209 are reportedly associated with cancer as discussed further below. LYVE1 is a type I integral membrane glycoprotein (24) which acts as a receptor, binding to both soluble and immobilized hyaluronan (HA), and may also be involved in lymphatic HA transport and lymph angiogenesis $(25,26)$. In 1999, Banerji et al (24) were the first to reveal that LYVE1 is a lymph-specific HA receptor and a unique marker for lymph vessels. Subsequently, Bono et al (27) demonstrated that a high LYVE1-positive lymphatic vessel number was associated with poor outcome in patients with ductal BC (28). The present study supports this conclusion, defining LYVE1 as a risk factor, and with upregulated expression increasing the risk score and the likelihood of poor prognosis. It was concluded that LYVE1 was an essential protein in the lymph angiogenesis and tumor 


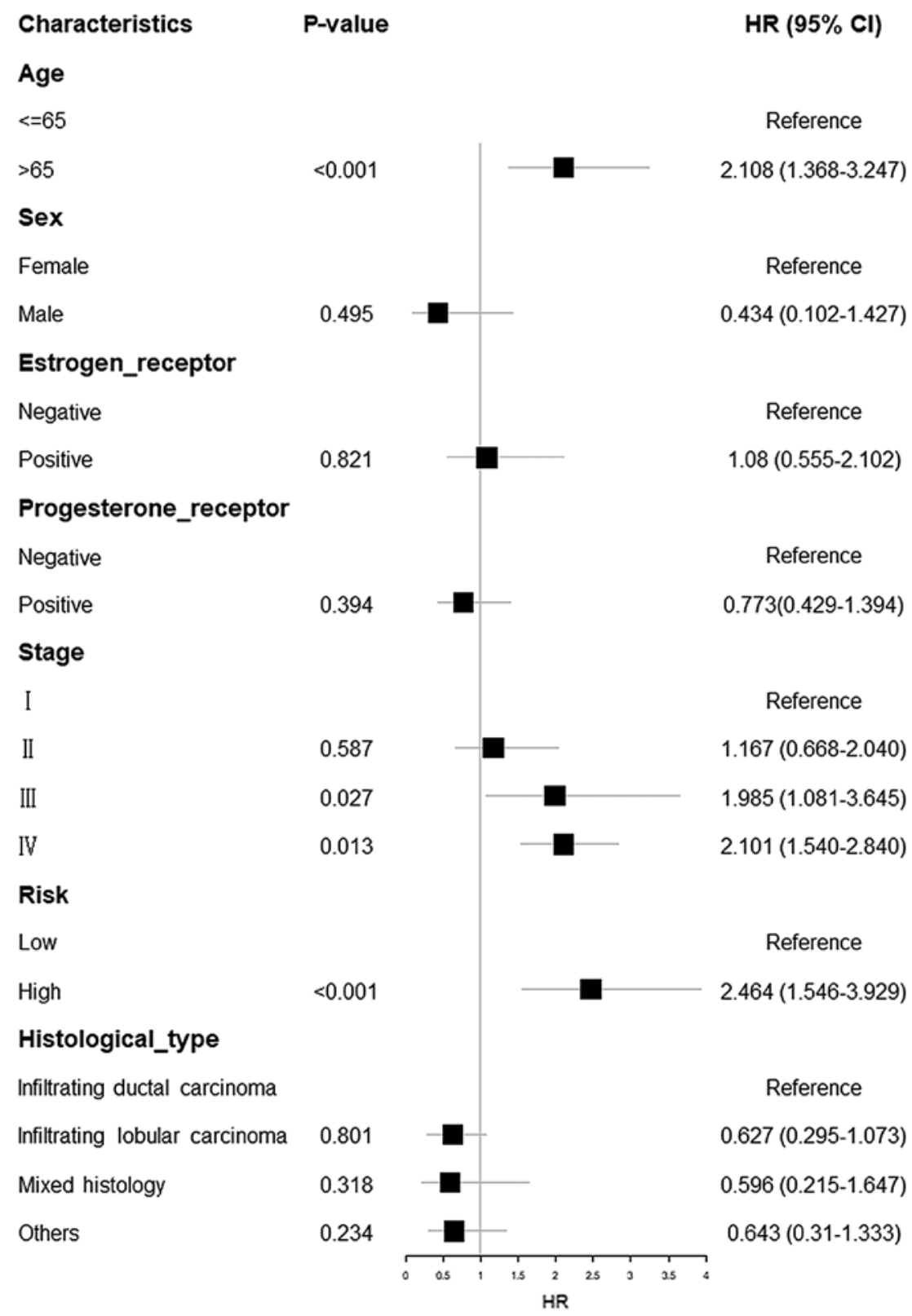

Figure 5. Clinical significance of clinical information and risk score in entire data set. HR, hazard ratio; CI, confidence interval.

metastasis of $\mathrm{BC}$, and that it may be a favorable candidate for targeted treatment. Furthermore, CD209 is a C-type lectin receptor expressed on the surface of macrophages and dendritic cells (DCs) (27). In the present study, CD209 expression was identified as a protective biomarker in the prognosis of BC. This conclusion was supported by van Gisbergen et al (29), who found revealed that the binding of SKBR3 cells to immature DCs was inhibited by CD209-resistant antibodies, thereby inhibiting the maturation of DCs and promoting tumor cell immune escape. Nevertheless, the functions of the other 5 genes are not currently known, and thus there is a requirement for further investigation. Using Cox regression analysis, a risk score model merging the aforementioned genes was established and may aid to predict the survival of patients with BC.

Although the 7-gene signature effectively predicted the outcome of patients with $\mathrm{BC}$, there are certain limitations to the present study. The risk score model was developed based on TCGA datasets and future studies require its validation in additional patient cohorts. However, due to a lack of data from patients in this age range, a reliable model of the younger subgroup could not be established. Furthermore, the treatment method serves an important role in disease prognosis, and the inclusion of treatment data in these analyses would increase the value of the subsequent results. However, there was insufficient data on the patients' treatment programs in the datasets, which was a major limitation and will be addressed in the collection of subsequent clinical data.

In conclusion, the 7-gene signature established in the present study was effective and stable in $\mathrm{BC}$ samples acquired from TCGA. Additional analysis indicated that the 7-gene signature functioned as an autonomous element for the prognosis of patients with BC. The results may potentially contribute to the development of more effective prognostic tools, and ultimately improve patient outcome. 
A

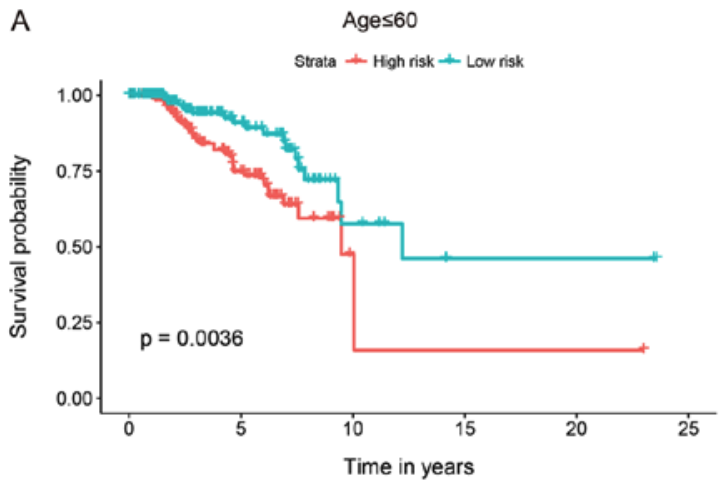

B

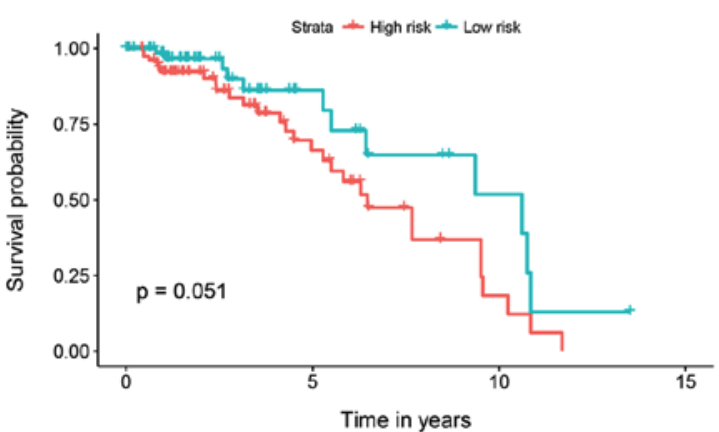

C

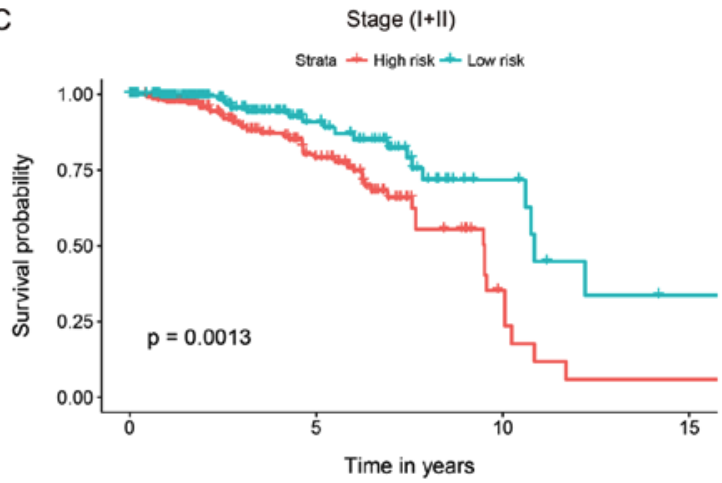

D

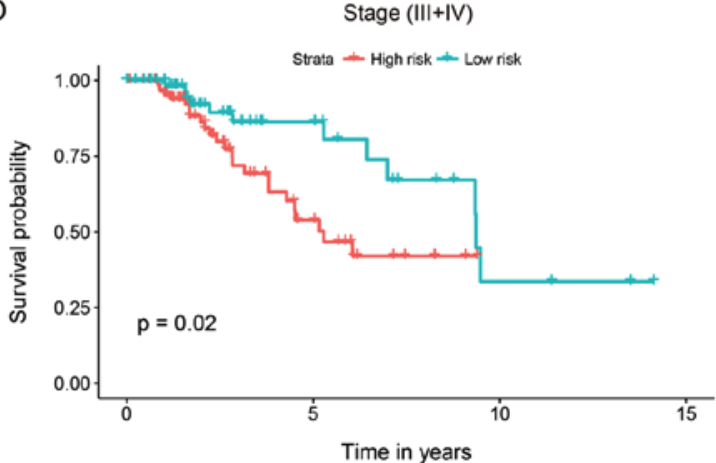

Figure 6. Kaplan-Meier survival curve analysis for overall survival of patients stratified by age and primary tumor stage using the 7-gene signature in the entire data set. (A) Kaplan-Meier tumor survival curves of the younger group $(\mathrm{n}=388)$. (B) Kaplan-Meier survival curves of the elder group ( $\mathrm{n}=256$ ). (C) Kaplan-Meier survival curves of the earlier-stage group $(n=399)$. (D) Kaplan-Meier survival curves of the advanced-stage group $(n=145)$.

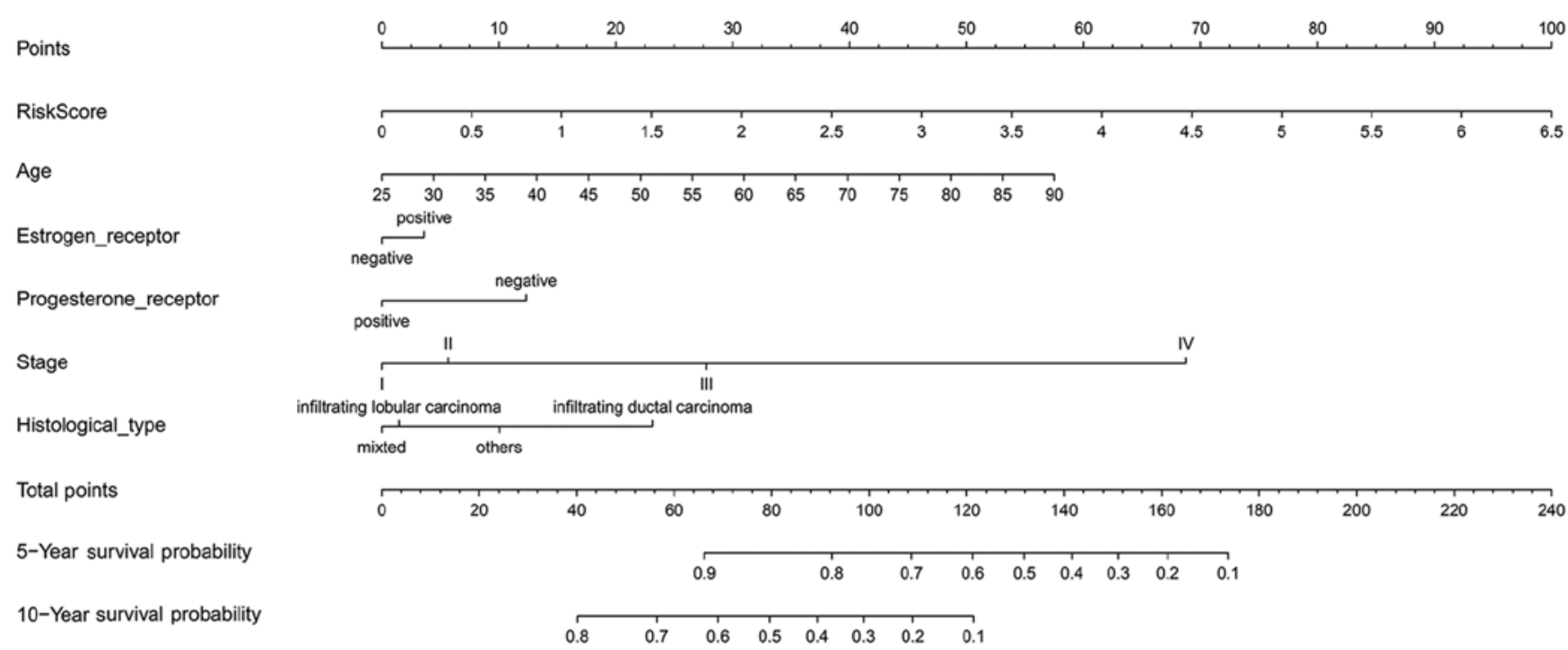

Figure 7. Nomogram containing clinical factors and risk score. Points represent the score of each variable, and Total points represents the cumulative score of each variable.

\section{Acknowledgements}

Not applicable.

\section{Funding}

The authors acknowledge the support received from the National Natural Science Foundation of China (grant no. 81560526), the Natural Science Foundation of Guang Xi (grant no. 2014GXNSFCA118011) and the College Young and Middle-Aged Teachers' Basic Ability Improvement Project of Guang Xi (grant no. 2017KY0484).

\section{Availability of data and materials}

The datasets used and/or analyzed during the current study are available from the corresponding author on reasonable request. 


\section{Authors' contributions}

WP and HL designed the experiment, provided financial support, revised the manuscript and gave final approval of the version to be published. LL and ZC performed the statistical analysis and wrote the paper. WS made substantive contributions to the work, including data collecting and manuscript revising.

\section{Ethics approval and consent to participate}

Not applicable.

\section{Patient consent for publication}

Not applicable.

\section{Competing interests}

The authors declare that they have no competing interests.

\section{References}

1. Gewefel H and Salhia B: Breast cancer in adolescent and young adult women. Clin Breast Cancer 14: 390-395, 2014.

2. Clough KB, Kaufman GJ, Nos C, Buccimazza I and Sarfati IM: Reply to comments on: Improving breast cancer surgery: A classification and quadrant per quadrant atlas for oncoplastic surgery. Ann Surg Oncol 18: 259-260, 2011.

3. Graham PJ, Brar MS, Foster T, McCall M, Bouchard-Fortier A, Temple W and Quan ML: Neoadjuvant chemotherapy for breast cancer, is practice changing? A population-based review of current surgical trends. Ann Surg Oncol 22: 3376-3382, 2015.

4. Dai D, Jin $H$ and Wang X: Nomogram for predicting survival in triple-negative breast cancer patients with histology of infiltrating duct carcinoma: A population-based study. Am J Cancer Res 8: 1576-1585, 2018.

5. Lee SK, Yang JH, Woo SY, Lee JE and Nam SJ: Nomogram for predicting invasion in patients with a preoperative diagnosis of ductal carcinoma in situ of the breast. Br J Surg 100: 1756-1763, 2013.

6. Balachandran VP, Gonen M, Smith JJ and Dematteo RP: Nomograms in oncology: More than meets the eye. Lancet Oncol 16: e173-e180, 2015.

7. Mortazavi A, Williams BA, McCue K, Schaeffer L and Wold B: Mapping and quantifying mammalian transcriptomes by RNA-Seq. Nat Methods 5: 621-628, 2008.

8. Han Y, Xu GX, Lu H, Yu DH, Ren Y, Wang L, Huang XH, Hou WJ, Wei ZH, Chen YP, et al: Dysregulation of miRNA-21 and their potential as biomarkers for the diagnosis of cervical cancer. Int J Clin Exp Pathol 8: 7131-7139, 2015.

9. Lee E, Collazolorduy A, Castillomartin M, Gong Y, Wang L, Oh WK, Galsky MD, Cordon-Cardo C and Zhu J: Identification of microR-106b as a prognostic biomarker of p53-like bladder cancers by ActMiR. Oncogene 37: 5858-5872, 2018.

10. Yue X, Lan F, Hu M,Pan Q, Wang Q and Wang J: Downregulation of serum microRNA-205 as a potential diagnostic and prognostic biomarker for human glioma. J Neurosurg 124: 122-128, 2016.

11. Lelo A, Prip F, Harris BT, Solomon D, Berry DL, Chaldekas K, Kumar A, Simko J, Jensen JB, Bhattacharyya P, et al: STAG2 is a biomarker for prediction of recurrence and progression in papillary non-muscle-invasive bladder cancer. Clin Cancer Res 24: 4145-4153, 2018

12. Feng A, Tu Z and Yin B: The effect of HMGB1 on the clinicopathological and prognostic features of non-small cell lung cancer. Oncotarget 7: 20507-20519, 2016.
13. Peng F, Shi X, Meng Y, Dong B, Xu G, Hou T, Shi Y and Liu T: Long non-coding RNA HOTTIP is upregulated in renal cell carcinoma and regulates cell growth and apoptosis by epigenetically silencing of LATS2. Biomed Pharmacother 105: 1133-1140, 2018.

14. Zhang L, Song X, Wang X, Xie Y, Wang Z, Xu Y, You X, Liang $\mathrm{Z}$ and Cao H: Circulating DNA of HOTAIR in serum is a novel biomarker for breast cancer. Breast Cancer Res Treat 152: 199-208, 2015.

15. Salomaa V, Havulinna A, Saarela O, Zeller T, Jousilahti $P$, Jula A, Muenzel T, Aromaa A, Evans A, Kuulasmaa K and Blankenberg S: Thirty-one novel biomarkers as predictors for clinically incident diabetes. PLoS One 5: e10100, 2010.

16. van de Vijver MJ, He YD, van't Veer LJ, Dai H, Hart AA, Voskuil DW, Schreiber GJ, Peterse JL, Roberts C, Marton MJ, et al: A gene-expression signature as a predictor of survival in breast cancer. N Engl J Med 347: 1999-2009, 2002.

17. Paik S, Tang G, Shak S, Kim C, Baker J, Kim W, Cronin M, Baehner FL, Watson D, Bryant J, et al: Gene expression and benefit of chemotherapy in women with node-negative, estrogen receptor-positive breast cancer. J Clin Oncol 24: 3726-3734, 2006.

18. Wang M, Klevebring D, Lindberg J, Czene K, Grönberg H and Rantalainen M: Determining breast cancer histological grade from RNA-sequencing data. Breast Cancer Res 18: 48, 2016.

19. Heagerty PJ, Lumley T and Pepe MS: Time-dependent ROC curves for censored survival data and a diagnostic marker. Biometrics 56: 337-344, 2000.

20. Harrell FE, Califf RM, Pryor DB, Lee KL and Rosati RA: Evaluating the yield of medical tests. JAMA 247: 2543-2546, 1982.

21. Zhao Y, Yang Y, Dai J, Xing D and Dong Y: IMPDH2 is highly expressed in breast cancer and predicts unfavourable prognosis. Biomarkers: Jul 2: 2018 (Epub ahead of print). doi: 10.1080/1354 750X.2018.1496360.

22. He K and Wang P: Unregulated long non-coding RNA-AK058003 promotes the proliferation, invasion and metastasis of breast cancer by regulating the expression levels of the $\gamma$-synuclein gene. Exp Ther Med 9: 1727-1732, 2015.

23. Guo J, Liu C, Wang W, Liu Y, He H, Chen C, Xiang R and Luo Y: Identification of serum miR-1915-3p and miR-455-3p as biomarkers for breast cancer. PLoS One 13: e0200716, 2018.

24. Banerji S, Ni J, Wang SX, Clasper S, Su J, Tammi R, Jones M and Jackson DG: LYVE-1, a new homologue of the CD44 glycoprotein, is a lymph-specific receptor for hyaluronan. J Cell Biol 144: 789-801, 1999.

25. Mattila MM, Ruohola JK, Karpanen T, Jackson DG, Alitalo K and Härkönen PL: VEGF-C induced lymphangiogenesis is associated with lymph node metastasis in orthotopic MCF-7 tumors. Int J Cancer 98: 946-951, 2002.

26. Skobe M, Hawighorst T, Jackson DG, Prevo R, Janes L, Velasco P, Riccardi L, Alitalo K, Claffey K and Detmar M: Induction of tumor lymphangiogenesis by VEGF-C promotes breast cancer metastasis. Nat Med 7: 192-198, 2001.

27. Bono P, Wasenius VM, Heikkilä P, Lundin J, Jackson DG and Joensuu H: High LYVE-1-positive lymphatic vessel numbers are associated with poor outcome in breast cancer. Clin Cancer Res 10: 7144-7149, 2004.

28. Mcgreal EP, Miller JL and Gordon S: Ligand recognition by antigen-presenting cell C-type lectin receptors. Curr Opin Immunol 17: 18-24, 2005.

29. van Gisbergen KP, Aarnoudse CA, Meijer GA, Geijtenbeek TB and Van Kooyk Y: Dendritic cells recognize tumor-specific glycosylation of carcinoembryonic antigen on colorectal cancer cells through dendritic cell-specific intercellular adhesion molecule-3-grabbing nonintegrin. Cancer Res 65: 5935-5944, 2005. 